EDITORIAL

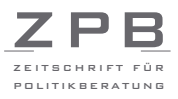

\title{
Does Strategy matter?
}

Joachim Raschke definiert in einem vielbeachteten politikwissenschaftlichen Grundlagenaufsatz Strategie als ,situationsübergreifende, erfolgsorientierte Ziel-Mittel-Umwelt-Kalküle“. Diese Definition deutet bereits an, dass Politikberatung im Bereich der Strategie ein komplexes Unterfangen ist und viele verschiedene Komponenten und $\mathrm{Zu}$ sammenhänge berücksichtigen muss. Dieser Vielfalt möchte die ZPB-Schwerpunktausgabe „Strategieberatung“ gerecht werden, indem sie Experten zu Wort kommen lässt, die in unterschiedlichsten Funktionen und Kontexten Strategieberatung betreiben oder analysieren.

Die vier wissenschaftlichen Beiträge können das Spektrum der Forschung im Bereich der politischen Strategieberatung naturgemäß nur andeuten. Gleichwohl bringen Sie unterschiedliche Ansätze und Perspektiven ein, die dem Leser einen Eindruck von den Konturen der Debatte vermitteln. Timo Grunden bewegt sich in seinem Beitrag über die Berater von Ministerpräsidenten im ,inner circle“ der Macht und auch Leonard Novy, Dominic Schwickert und Thomas Fischer fokussieren auf die politischen Akteure, wenn sie Ideen zur Erhöhung der Strategiefähigkeit von Politikern und Parteien entwickeln. Die Aufsätze von Frank Nullmeier und Christopher Gohl hingegen versuchen eine theoretische Annäherung und greifen dabei unterschiedliche Aspekte heraus. Frank Nullmeier fasst strategische Kommunikationsberatung mit Hilfe des Veto-Spieler-Theorems während Christopher Gohl die prozedurale Komponente von Politik betont. Damit schließt er an das eingangs genannte Zitat von Joachim Raschke an, der Strategie als „situationsübergreifend“ charakterisiert.

Mit Volker Riegger, einem erfahrenen Wahlkampf-Manager, und Stephan Scholtissek, dem Deutschland-Chef und somit Chefstrategen eines großen Beratungshauses, eröffnen zwei führende Köpfe der deutschen Strategieberatung die Rubrik „Essays“. Hier werden unsere Autoren dazu ermuntert, jenseits üblicher wissenschaftlicher oder redaktioneller Konventionen in den freien Raum hinein zu denken. Die entstandenen Texte, so unterschiedlich sie auch erscheinen mögen, haben einen gemeinsamen Nenner: Sie wagen beide einen Blick in die Zukunft und deuten die großen kommenden Entwicklungslinien der Strategieberatung an.

Darüber hinaus bietet auch diese Ausgabe der ZPB eine große Auswahl an Beiträgen aus allen Teilen der Beratungswelt. In den Beratungsportraits werden der Chefberater von Barack Obama, David Axelrod, das international tätige Unternehmen Penn, Schoen and Berland Associates sowie der Studiengang „Politische Kommunikation“ in Düsseldorf vorgestellt. Einblicke in die Beratungspraxis geben Jan Ole Kiso, der am britischen Office of Climate Change arbeitet, sowie Manfred Mai mit einem Bericht über die Olympiabewerbung der Rhein-Ruhr-Region. Und im Forum skizzieren Manuela Glaab, Nikolaus Huss sowie Joachim Raschke und Ralf Tils ihre Ideen und Konzepte für eine strategische Politikberatung. Ergänzt werden diese Beiträge um Kurznachrichten in den Corners und den Literaturteil. Dieser trägt dem Wunsch nach einer Bestandsaufnah- 
me der umfangreichen Literatur im Bereich der Politikberatung Rechnung und umfasst Überblicke zur Nachrichtenlage und den einschlägigen Publikationen.

Unser Dank gilt allen Autorinnen und Autoren, den Mitarbeiterinnen und Mitarbeitern in Redaktion und Verlag sowie den Mitgliedern unseres Beirates. Wir freuen uns sehr über die ersten sichtbaren Erfolge unseres Projekts, die ohne die Unterstützung all dieser Personen nicht möglich gewesen wären. Zudem möchten uns an dieser Stelle auch sehr herzlich für die zahlreichen Anregungen bedanken, die Leserinnen und Leser an uns herangetragen haben. Das positive Feedback und die interessanten Ideen bestärken uns in unserem Ziel, die „Zeitschrift für Politikberatung“ kontinuierlich zu verbessern und zu einem führenden Organ der Analysen und Debatten im Bereich der Politikberatung zu machen.

Svenja Falk, Dieter Rehfeld, Andrea Römmele, Martin Thunert und Henrik Schober 